\title{
Rough or Smooth Balls? That is the Question
}

\author{
T.G. Myers ${ }^{1} \dagger$, and S.L. MitchelL ${ }^{2}$ \\ ${ }^{1}$ Centre de Recerca Matemàtica, Barcelona \\ ${ }^{2}$ University of Limerick \\ (Communicated to MIIR on 2 April 2021)
}

Study Group: MISGSA2011, 10-14 January, 2011, University of the Witwatersrand, Johannesburg, South Africa.

Communicated by: David P. Mason

Industrial Partner: University of Witwatersrand Football Club

Presenter: Dennis Tshabalala

Team Members: A.G. Fareo, University of the Witwatersrand; D. Ikpe, University of the Witwatersrand; J. Manyisa, University of the Witwatersrand; S.L. Mitchell, University of Limerick; G. Muchatibaya, Cape Peninsula University of Technology; T.G. Myers, Centre de Recerca Matemàtica, Barcelona; L. Ndlovu, University of the Witwatersrand; C. Please, University of Oxford; D. Rikhotso, University of the Witwatersrand.

Industrial Sector: Sports

Tools: Fluid dynamics, asymptotics

Key Words: sports science, ball games,

MSC2020 Codes: 35, 34

$\dagger$ Corresponding Author. tmyers@crm.cat 


\title{
ROUGH OR SMOOTH BALLS? THAT IS THE QUESTION
}

\author{
T.G. Myers*, S.L. Mitchell ${ }^{\dagger}$ \\ Study group participants \\ T.G. Myers, S.L. Mitchell, C. Please \\ G. Muchatibaya, J. Manyisa, D. Rikhotso, L. Ndlovu, \\ D. Ikpe, A.G. Fareo \\ Industry representative \\ Dennis Tshabalala
}

\begin{abstract}
The question posed at the study group, by a South African premiership team based in Johannesburg, was basically, 'Can we choose a match ball that will disadvantage opponents?'

In this report we answer the question through focussing on the motion of a football through the air (which is where the difference in the ball's behaviour due to altitude is greatest). The forces affecting a ball's motion are described and we show how this translates into a mathematical problem consisting of a system of coupled nonlinear differential equations. Numerical and approximate analytical solutions are presented and compared. We then show how the mathematical results may be used to guide the choice of ball.
\end{abstract}

\section{Introduction}

The problem posed at the study group was a simple one: given the different atmospheric conditions between Johannesburg and most other South African cities, can

${ }^{*}$ Centre de Recerca Mathemàtica, Campus de Bellaterra, Edifici C, 08193 Bellaterra, Barcelona, Spain.e-mail: tmyers@crm.cat

${ }^{\dagger}$ MACSI, Department of Mathematics and Statistics, University of Limerick, Limerick, Ireland. e-mail: sarah.mitchell@ul.ie 
we choose a match ball that will give an advantage to the home team? The choice of ball was in fact limited between balls supplied by the sponsor Nike, the T90, Tracer and Omni. The main obvious difference between the balls is that the T90 has a dimpled surface, making it relatively rough, whereas the Tracer and Omni have a shiny surface.

At the meeting we focussed on the motion of the ball in the air and in particular from a free kick or corner. One reason for this is that these are relatively controlled situations and there is much data on a ball's motion through the air. A second reason is that free kicks are an important factor in scoring: in the 1998 world cup 42 of the 171 goals scored came from set-plays, with $50 \%$ of these from free kicks [1]. Consequently understanding the ball motion through the air from a free kick or corner could provide important information concerning the best ball choice. Another reason is that Johannesburg is located high above sea level (at around $2000 \mathrm{~m}$ ). The air density is approximately $20 \%$ below that at sea level [5] and so we expect that the greatest difference in ball motion between Johannesburg and cities at lower altitude will be when it moves through the air.

As a football moves through the air it is subject to two main forces, gravity and air resistance or drag. The magnitude of the drag is (approximately) proportional to the square of the velocity and so the main effect of drag is to reduce the forward motion. However, it is also drag that causes the swerve. For example, if we give a ball top-spin then the top of the ball moves forward (in the direction of the ball's motion) whilst the bottom moves backwards. The top therefore has a higher forward velocity than the bottom. The higher velocity results in higher drag at the top than at the bottom (it also leads to a thinner boundary layer and earlier separation) with the result that the pressure due to drag is greater at the top than the bottom. This pressure difference then causes the ball to move downwards. Side spin will likewise lead to sideways swerve, towards the side with a lower velocity. The spin induced swerve is termed the Magnus effect. It is discussed in greater detail in [4]. Note, typically a ball is kicked at around $25 \mathrm{~m} / \mathrm{s}$ resulting in a relatively predictable swerve. However, at low speeds, around $10-15 \mathrm{~m} / \mathrm{s}$, there is a transition in the air flow around the ball which may lead to the "reverse Magnus" effect. The transition speed depends crucially on the ball and is affected by factors such as surface roughness, pattern and stitching.

Given that the main focus of this report is on the effect of spin and its role in choice of ball we note that there is no qualitative difference between top spin and side spin. That is, if we study only side spin the conclusions will be equally valid for top spin. This observation allows us to confine our attention solely to two-dimensional motion. 


\section{Mathematical Model}

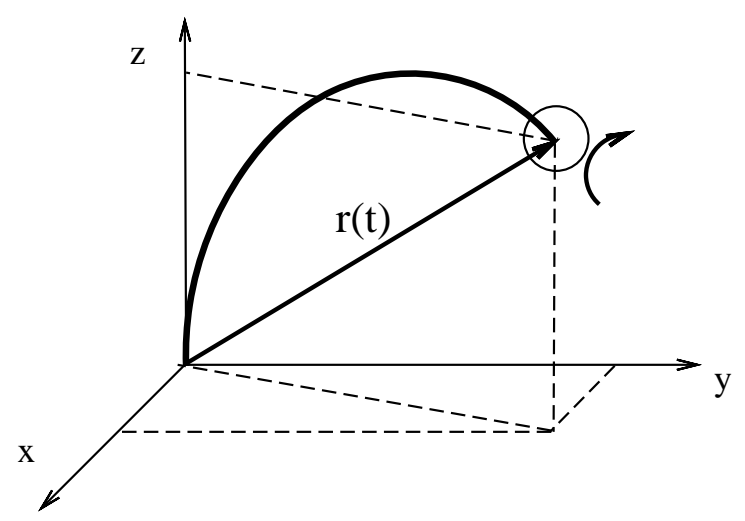

Figure 1: Trajectory of a soccer ball.

Consider a ball initially at the origin, $\mathbf{r}(0)=\mathbf{0}$, which is kicked primarily in the $y$ direction. The ball follows a trajectory denoted by $\mathbf{r}(t)$, as shown in Figure 1 . For simplicity we assume that the spin only occurs about an axis contained in the $x-z$ plane. Note we could easily generalise this, following the equations given in [3], however, with the aim of simply finding how a given ball swerves it is sufficient to analyse the simpler model with swerve in a single plane.

If we distinguish between the forward drag and Magnus (lift) effect, using the subscript notation $d$ and $l$ for the drag and lift, then the total force $\mathbf{F}$ acting on the ball is

$$
\mathbf{F}=m \mathbf{g}+\mathbf{F}_{d}+\mathbf{F}_{l}
$$

where

$$
\mathbf{F}_{d}=-\frac{1}{2} \rho A|\boldsymbol{v}|^{2} C_{d} \hat{\boldsymbol{v}}, \quad \mathbf{F}_{l}=\frac{1}{2} \rho A|\boldsymbol{v}|^{2} C_{l} \hat{\boldsymbol{\sigma}} \times \hat{\boldsymbol{v}} .
$$

Here $m$ is the mass of the ball, $A$ is its cross-sectional area, $\rho$ is the density of air,

$$
|\hat{\boldsymbol{v}}|=\sqrt{\left(\dot{x}^{2}+\dot{y}^{2}+\dot{z}^{2}\right)},
$$

is the magnitude of the velocity vector, $C_{d}$ and $C_{l}$ are the experimentally determined drag and lift coefficients (which depend on the ball design). The unit vector $\hat{\boldsymbol{v}}$ defines the direction of $\boldsymbol{v}$

$$
\hat{\boldsymbol{v}}=\cos \psi \sin \theta \mathbf{i}+\cos \psi \cos \theta \mathbf{j}+\sin \psi \mathbf{k} .
$$

Note, we define angles according to the convention of $[1,7]$ (which is essentially opposite to normal convention). The angle $\theta$ is measured from the $y$ axis to the 
projection of the velocity vector onto the $x-y$ plane. The angle $\psi$ is the angle of inclination in the $z$ direction measured from the $x-y$ plane. From the definition of $\hat{\boldsymbol{v}}$ we may express the angles in terms of the Cartesian velocity components

$$
\cos \psi \sin \theta=\frac{\dot{x}}{|\boldsymbol{v}|}, \quad \cos \psi \cos \theta=\frac{\dot{y}}{|\boldsymbol{v}|}, \quad \sin \psi=\frac{\dot{z}}{|\boldsymbol{v}|} .
$$

The spin axis is

$$
\boldsymbol{\sigma}=\cos \gamma \mathbf{i}-\sin \gamma \mathbf{k}
$$

where $\gamma$ is the angle between the negative $x$ axis and the $z$ axis. In keeping with previous studies we assume that the orientation of the spin axis and spin rate are constant. In fact the spin rate will decrease very slowly, see [5], but this will not affect the conclusions of our model.

The differential equation for the flight is, therefore

$$
\ddot{\mathbf{r}}=\mathbf{g}-k_{d}|\boldsymbol{v}|^{2} \hat{\boldsymbol{v}}+k_{l}|\boldsymbol{v}|^{2} \boldsymbol{\sigma} \times \hat{\boldsymbol{v}},
$$

where $k_{d}=\rho A C_{d} / 2 m$ and $k_{l}=\rho A C_{l} / 2 m$. In component form this is

$$
\begin{aligned}
& \ddot{x}=-|\boldsymbol{v}|\left\{k_{d} \dot{x}-k_{l} \sin \gamma \dot{y}\right\} \\
& \ddot{y}=-|\boldsymbol{v}|\left\{k_{d} \dot{y}+k_{l}[\cos \gamma \dot{z}+\sin \gamma \dot{x}]\right\} \\
& \ddot{z}=-g-|\boldsymbol{v}|\left\{k_{d} \dot{z}-k_{l} \cos \gamma \dot{y}\right\} .
\end{aligned}
$$

This is the basic set of equations governing the motion of a football. The appearance of $|\boldsymbol{v}|$ means that they are nonlinear and difficult to solve analytically. Bray \& Kerwin [1] note that they have no closed form solution and so must be solved numerically.

Given that our focus is on the effect of spin, it is sufficient to deal with only a two-dimensional model. Any conclusions concerning ball choice in two-dimensions will hold in three-dimensions. Hence in the following sections we will study the simpler model defined by

$$
\begin{aligned}
& \ddot{x}=-|\boldsymbol{v}|\left\{k_{d} \dot{x}-k_{l} \sin \gamma \dot{y}\right\} \\
& \ddot{y}=-|\boldsymbol{v}|\left\{k_{d} \dot{y}+k_{l} \sin \gamma \dot{x}\right\},
\end{aligned}
$$

where now $|\boldsymbol{v}|=\sqrt{\left(\dot{x}^{2}+\dot{y}^{2}\right)}$. This means that we are restricting the ball motion to move primarily in the direction of the kick, the $y$ direction, and any swerve is caused by side-spin (and so we must choose $\gamma \approx \pi / 2$ to ensure there is a significant spin component). To highlight the effect of spin further we impose the following initial conditions

$$
x(0)=y(0)=0, \quad \dot{x}(0)=0, \quad \dot{y}(0)=v .
$$


That is, the ball is initially placed at the origin of the system and kicked purely in the $y$ direction. There is no initial $x$ velocity and so any motion in this direction must come from the spin of the ball.

In the following section we will reduce this system using a simple dominant balance argument, which then allows us to find an analytical solution. Subsequently we will formalise the reduction, via non-dimensionalisation, and then find a more accurate perturbation solution. This approach is easily extended to three-dimensions, hence, although the comment of Bray \& Kerwin [1] that there is no closed form solution is correct, it is possible to find an accurate approximation to the system and so remove the need for a numerical solution.

\section{Approximate solution method}

Even the two-dimensional equations $(11,12)$ contain a number of terms that make the system difficult to treat analytically. However, it is apparent that some will have a greater effect than others. For example, since the initial kick is predominantly in the $y$ direction then clearly $\dot{y} \gg \dot{x}$ and so $v \approx \dot{y}$. The coefficients $C_{d}, C_{l}=\mathcal{O}(0.3)$ and so $k_{d}, k_{l} \approx 0.01$. Then if we consider the $x$ equation, provided the spin axis is such that $\gamma$ is not close to zero, it follows that $k_{d} \dot{x} \ll k_{l} \sin \gamma \dot{y}$. In the $y$ direction obviously the drag is much greater than the lift implying that $k_{d} \dot{y} \gg k_{l} \sin \gamma \dot{x}$. Consequently the governing equations must be well approximated by the system

$$
\ddot{x}=k_{l} \sin \gamma \dot{y}^{2}, \quad \ddot{y}=-k_{d} \dot{y}^{2} .
$$

This system can be solved analytically. Before writing down the solution we first note that the $x$ equation may be expressed as

$$
\ddot{x}=-\frac{k_{l}}{k_{d}} \sin \gamma \ddot{y} .
$$

Denoting the initial conditions as $(x, y)=\mathbf{0},(\dot{x}, \dot{y})=(0, v)$ at $t=0$ then we first integrate the $y$ equation to find

$$
y=\frac{1}{k_{d}} \ln \left(1+k_{d} v t\right) .
$$

Substituting this into the $x$ equation gives

$$
x=-\frac{k_{l}}{k_{d}} \sin \gamma(y-v t) .
$$

This solution highlights the dominant factors that determine the position of the ball, that is, from the $y$ equation it is clear that only the drag, $k_{d}$, and initial velocity 
determine the position with respect to time. From the $x$ equation we may conclude that the amount of swerve depends on $v, k_{d}, k_{l}$ and also the spin angle. Equations $(16,17)$ provide a first approximation to the solution of the system and indicate that while the $y$ motion is relatively simple, the swerve (in the $x$ direction) depends on more parameters. We will now look into a more formal method to approximate the ball motion and so improve on the above equations.

\section{Perturbation solution}

We can non-dimensionalise the system by setting

$$
\hat{x}=\frac{x}{L_{1}}, \quad \hat{y}=\frac{y}{L_{2}}, \quad \hat{t}=\frac{t}{\tau} .
$$

The length-scale $L_{2}$ is simply the distance of the free kick, that is, the distance between the ball and goal, which we will take to be $20 \mathrm{~m}$. Note, another distance can be found from the governing equations $L_{2}=1 / k_{d} \approx 100 \mathrm{~m}$. This is the lengthscale over which drag will cause the ball to stop. Since the free kick is taken over a much shorter distance we will take $L_{2}=20 \mathrm{~m}$. The time-scale $\tau$ then comes from the time taken for the ball to travel the distance $L_{2}$. If the initial kick velocity is $v$ then $\tau=L_{2} / v \mathrm{~s}$. For example, if $v=20 \mathrm{~m} / \mathrm{s}$ then $\tau=1 \mathrm{~s}$ is approximately the time taken for the ball to reach the goal. The length-scale $L_{1}$ is, as yet, unknown.

Now consider the velocity vector $|\boldsymbol{v}|$. In non-dimensional form

$$
|\hat{\boldsymbol{v}}|=\sqrt{\frac{L_{1}^{2}}{\tau^{2}} \dot{\hat{x}}^{2}+\frac{L_{2}^{2}}{\tau^{2}} \dot{\hat{y}}^{2}}=\frac{L_{2}}{\tau} \dot{\hat{y}} \sqrt{1+\frac{L_{1}^{2}}{L_{2}^{2}} \frac{\dot{\hat{x}}^{2}}{\dot{\hat{y}}^{2}}} .
$$

The $x$ equation becomes

$$
\frac{L_{1}}{\tau^{2}} \ddot{\hat{x}}=-\frac{L_{2}}{\tau} \dot{\hat{y}} \sqrt{1+\frac{L_{1}^{2}}{L_{2}^{2}} \frac{\dot{\hat{x}}^{2}}{\dot{\hat{y}}^{2}}}\left(k_{d} \frac{L_{1}}{\tau} \dot{\hat{x}}-k_{l} \sin \gamma \frac{L_{2}}{\tau} \dot{\hat{y}}\right) .
$$

Hence

$$
\ddot{\hat{x}}=-\dot{\hat{y}} \sqrt{1+\frac{L_{1}^{2}}{L_{2}^{2}} \frac{\dot{\hat{x}}^{2}}{\dot{\hat{y}}^{2}}}\left(k_{d} L_{2} \dot{\hat{x}}-k_{l} \sin \gamma \frac{L_{2}^{2}}{L_{1}} \dot{\hat{y}}\right) .
$$

As stated previously, the motion in the $x$-direction comes from the side-spin term and this indicates the length-scale $L_{1}=k_{l} \sin \gamma L_{2}^{2}$. If we set $k_{d}=0.013, k_{l}=0.01$, $\gamma=\pi / 2, L_{2}=20$ then we see $L_{1}=5.2 \mathrm{~m}$, meaning that we expect the ball to swerve $\mathcal{O}(5) \mathrm{m}$ during the flight. We now denote $\epsilon=k_{d} L_{2}(\approx 0.3$ for the given parameter 
values). Noting that $L_{1} / L_{2}=5.2 / 20$ we may set $L_{1} / L_{2}=a \epsilon$. A similar analysis reduces the $y$ equation to

$$
\ddot{\hat{y}}=-\dot{\hat{y}} \sqrt{1+\frac{L_{1}^{2}}{L_{2}^{2}} \frac{\dot{\hat{x}}^{2}}{\dot{\hat{y}}^{2}}}\left(k_{d} L_{2} \dot{\hat{y}}+k_{l} \sin \gamma L_{1} \dot{\hat{x}}\right) .
$$

The final term on the right hand side $k_{l} \sin \gamma L_{1} \approx 0.04$. Noting that $\epsilon^{2}=0.068$ we denote $k_{l} \sin \gamma L_{1}=b \epsilon^{2}$. Dropping the hats we may finally write our system as

$$
\begin{gathered}
\ddot{x}=-\dot{y} \sqrt{1+a^{2} \epsilon^{2} \frac{\dot{x}^{2}}{\dot{y}^{2}}}(\epsilon \dot{x}-\dot{y}), \quad \ddot{y}=-\dot{y} \sqrt{1+a^{2} \epsilon^{2} \frac{\dot{x}^{2}}{\dot{y}^{2}}}\left(\epsilon \dot{y}+b \epsilon^{2} \dot{x}\right), \\
x(0)=y(0)=0, \quad \dot{x}(0)=0, \quad \dot{y}(0)=1 .
\end{gathered}
$$

Now we carry out a standard perturbation analysis, setting

$$
x=x_{0}+\epsilon x_{1}+\epsilon^{2} x_{2}+\epsilon^{2} x_{3}+\cdots, \quad y=y_{0}+\epsilon y_{1}+\epsilon^{2} y_{2}+\epsilon^{3} y_{3}+\cdots .
$$

Substituting these expansions into the equations in (23) leads to

$$
\begin{aligned}
& y=t-\epsilon \frac{t^{2}}{2}+\epsilon^{2}(2-b) \frac{t^{3}}{6}-\epsilon^{3}\left(6+a^{2}-7 b\right) \frac{t^{4}}{24}+\mathcal{O}\left(\epsilon^{4}\right) \\
& x=\frac{t^{2}}{2}-\epsilon \frac{t^{3}}{2}+\epsilon^{2}\left(11+a^{2}-2 b\right) \frac{t^{4}}{24}-\epsilon^{3}\left(50+15 a^{2}-25 b\right) \frac{t^{5}}{120}+\mathcal{O}\left(\epsilon^{4}\right) .
\end{aligned}
$$

Returning to dimensional form, by setting $x \rightarrow x / L_{1}=x /\left(k_{l} L_{2}^{2} \sin \gamma\right), y \rightarrow$ $y / L_{2}, t \rightarrow t v / L_{2}$, we obtain

$$
\begin{aligned}
& x=k_{l} \sin \gamma \frac{v^{2} t^{2}}{2}\left(1-k_{d} v t+\left(11+a^{2}-2 b\right) \frac{\left(k_{d} v t\right)^{2}}{12}-\left(50+15 a^{2}-25 b\right) \frac{\left(k_{d} v t\right)^{3}}{60}\right), \\
& y=v t\left(1-k_{d} v \frac{t}{2}+(2-b) \frac{\left(k_{d} v t\right)^{2}}{6}-\left(6+a^{2}-7 b\right) \frac{\left(k_{d} v t\right)^{3}}{24}\right) .
\end{aligned}
$$

The $y$ equation shows that the distance travelled is approximately proportional to the initial velocity and time but that drag reduces this and the effect of drag increases with time. However, since swerve due to side-spin is our main interest the $x$ equation is the most revealing. From this we see that to leading order the distance travelled in the $x$ direction is proportional to the drag coefficient $k_{l}$ and also $(v t)^{2}$. The ball design and atmospheric conditions contribute through $k_{l}$. The product $v t$ is the first approximation to the distance travelled in the $y$ direction. The quadratic 
dependence then indicates the importance of taking a kick far from the goal. The further the ball travels the more it will swerve (this is obviously well known to free kick specialists in football who frequently attempt to move the ball away from the goal). Perhaps the most famous example is the free-kick of Roberto Carlos against France in 1997 which exhibited spectacular curve at the end of the flight. This free kick was taken approximately $35 \mathrm{~m}$ from goal, see YouTube or [2]. The forward drag $k_{d}$ enters at first order, that is, it's effect is relatively small (although it grows with time) and it acts to reduce the swerve.

Note, if we expand the solutions obtained by just retaining the dominant terms, equations $(16,17)$, for small $k_{d} v t$ then the $y$ expansion agrees with equation $(29)$ to $\mathcal{O}(\epsilon)$. The $x$ expansion only agrees at leading order. Hence the dominant term balance will provide a reasonable approximation to the $y$ motion but, except for at very small times, will not be so good in the $x$ direction. We will see this in the results shown later.

\section{Results}

Denoting the variables by

$$
x=f_{1}, \quad \dot{x}=f_{2}, \quad y=f_{3}, \quad \dot{y}=f_{4},
$$

the system represented by $(11,12)$ may be written as a set of first order differential equations

$$
\begin{array}{ll}
\dot{f}_{1}=f_{2}, & \dot{f}_{2}=-\sqrt{f_{2}^{2}+f_{4}^{2}}\left(k_{d} f_{2}-k_{l} \sin \gamma f_{4}\right), \\
\dot{f}_{3}=f_{4}, & \dot{f}_{4}=-\sqrt{f_{2}^{2}+f_{4}^{2}}\left(k_{d} f_{2}+k_{l} \sin \gamma f_{2}\right),
\end{array}
$$

subject to $f_{1}(0)=f_{2}(0)=f_{3}(0)=0, f_{4}(0)=v$. This system is easily solved numerically by MATLAB. In the following figures we compare the numerical solution with the approximate solutions of the previous two sections. Parameter values used in the calculations are provided in Table 1.

Figure 2 displays the different solutions for $x(t)$. The upper plot shows the numerical solution (solid line) and three perturbation solutions, to $\mathcal{O}\left(\epsilon, \epsilon^{2}, \epsilon^{3}\right)$. The $\mathcal{O}(\epsilon)$ solution is the dotted line lying below the numerical solution. The error at $t=1$ is $\left(x_{\text {num }}-x_{\mathcal{O}(\epsilon)}\right) / x_{\text {num }} \approx 0.08$, or $8 \%$ (this is in keeping with the order of neglected terms $\left.\mathcal{O}\left(\epsilon^{2}\right) \approx 0.09\right)$. The dash-dot line visible just above the numerical solution is the $\mathcal{O}\left(\epsilon^{2}\right)$ solution (with an expected error of $\mathcal{O}\left(\epsilon^{3}\right) \approx 0.003 \approx 0.3 \%$ ). The $\mathcal{O}\left(\epsilon^{3}\right)$ result is also plotted, as a dashed line, but this is indistinguishable from the numerical solution. The lower plot shows a comparison of the numerical and 

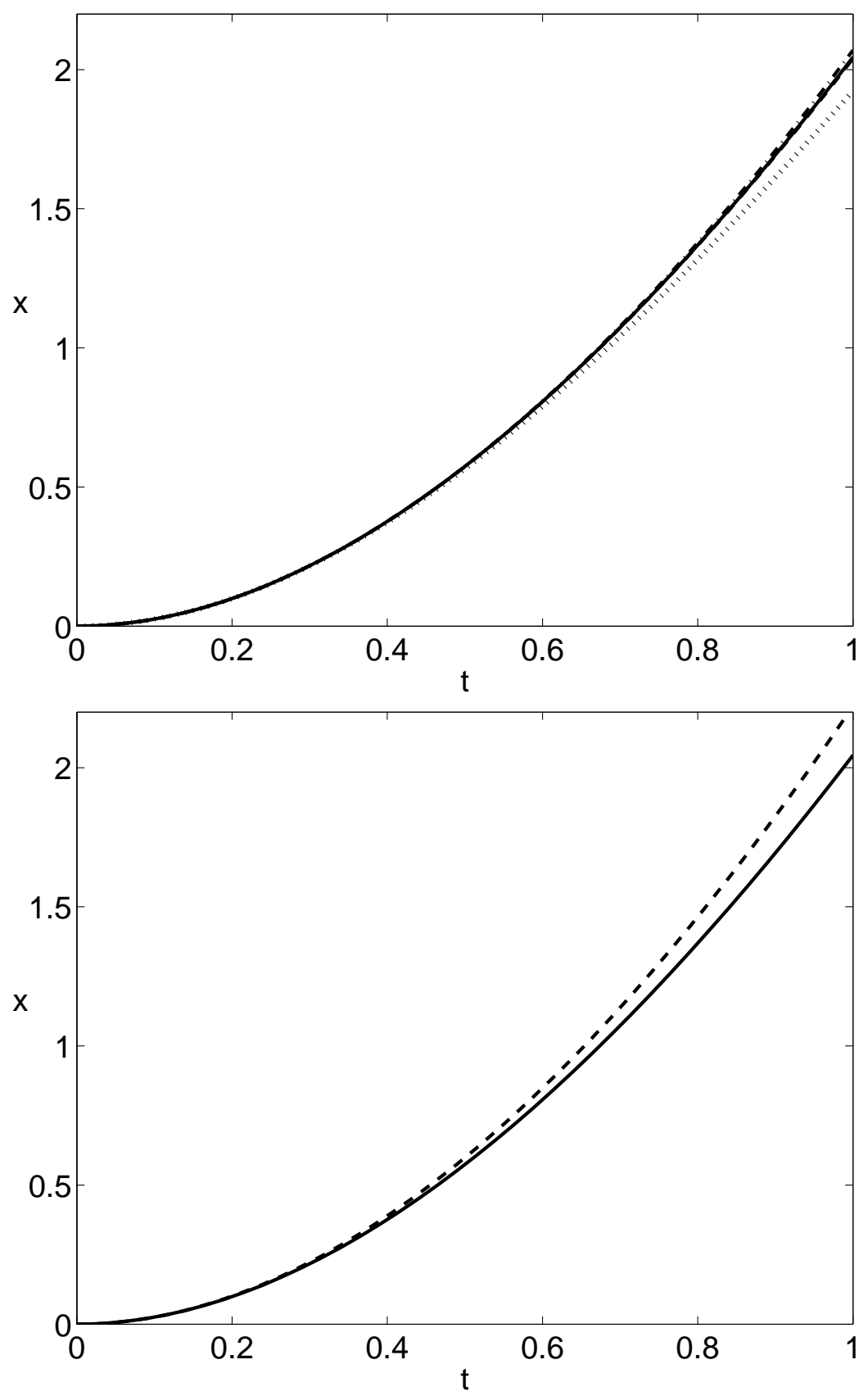

Figure 2: The $x$ position plotted against time: Upper plot shows the numerical solution (solid line) compared with the perturbation solutions $\left(\mathcal{O}(\epsilon)\right.$ is dotted, $\mathcal{O}\left(\epsilon^{2}\right)$ is dot-dashed and $\mathcal{O}\left(\epsilon^{3}\right)$ is dashed). Lower plot shows comparison of numerical solution (solid line) and the logarithmic solution (dashed). 


\begin{tabular}{|c|c|c|}
\hline Parameter & Value & Units \\
\hline$C_{d}$ & 0.35 & \\
$C_{l}$ & 0.27 & \\
$\gamma$ & 80 & degree \\
$m$ & 0.45 & $\mathrm{~kg}$ \\
$\rho$ & 1 & $\mathrm{~kg} \mathrm{~m}^{-3}$ \\
$r$ & 0.11 & $\mathrm{~m}$ \\
$A$ & 0.039 & $\mathrm{~m}^{2}$ \\
\hline
\end{tabular}

Table 1: Parameter values.

approximate solution, equation (17). Obviously this is much less accurate than the perturbation solution, but it still shows the correct qualitative behaviour.

Figure 3 shows the equivalent results for $y(t)$. Now, even at $\mathcal{O}(\epsilon)$ the perturbation solution is highly accurate. The approximate solution of equation (16) is also very close to the exact solution. The accuracy of the approximations $(16,17)$ is in line with the observations based on the expansions of the previous section.

Equations $(28,29)$ give explicit and accurate analytical representations for the position of the ball. Noting $a=k_{l} \sin \gamma / k_{d}$, it is clear that the main factors affecting the $y$ motion of the ball are the initial velocity $v$ and the drag coefficient $k_{d}$. The only contribution of the spin, through $k_{l}$ occurs at 3rd order and so is virtually negligible. That is, we can safely represent the motion in the $y$ direction by equation (14b) and for a given initial velocity if we wish to change the motion in the $y$ direction we must simply look into factors affecting $k_{d}$. Note, the terms in the $y$ series expansion also depend on $t$ and so it is possible that the lower order terms can dominate for sufficiently large $t$. If we consider when the first and second terms have the same magnitude we find $t=2 /\left(k_{d} v\right)=10 \mathrm{~s}$. Since this is too long a time-scale for a free kick we assume that our conclusions hold for all sensible times. The motion in the $x$ direction is dominated by the leading order term and so the swerve is primarily due to the size of $k_{l}, v, t, \gamma$. However, the presence of the $k_{d}$ term at first order means that this drag coefficient also plays an important role and its importance will increase in time. For example, at $t=0.1$ the first order term contributes around $1.5 \%$ of the movement whilst at $t=1$ it contributes $15 \%$. Practically this means that the swerve of the ball is controlled by a number of factors. To change the swerve we could look at changing $k_{l}, \gamma$ or to a lesser extent $k_{d}$. From the above results it is clear that the perturbation solutions are highly accurate. The beauty of calculating the solutions in this form, rather than numerically, is that we may see directly the effect of parameter values on the ball's motion. The $y$ solution shows that the motion is dominated by the initial kicking speed. At first order we find that the drag $k_{d}$ acts 

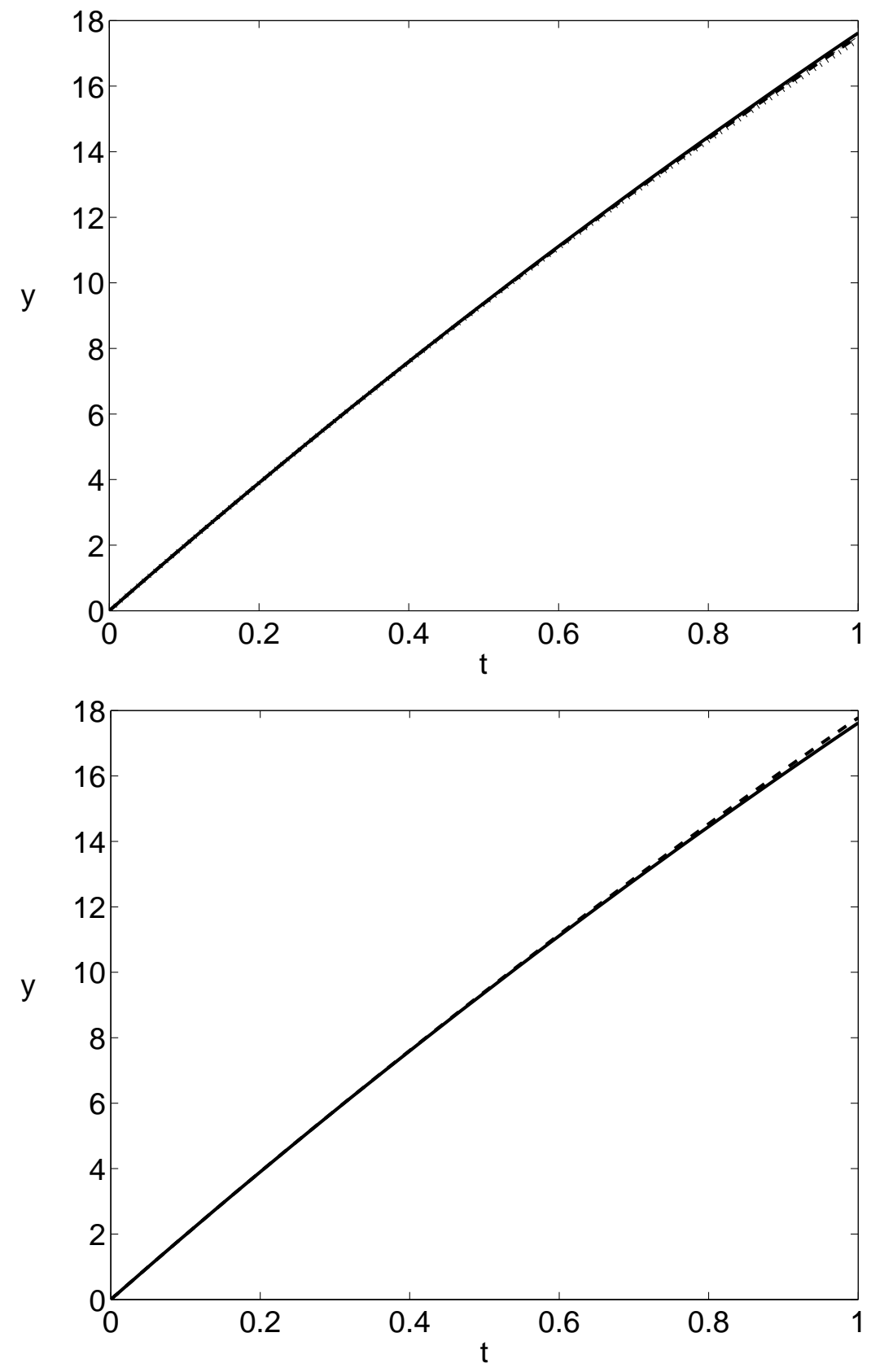

Figure 3: The $y$ position plotted against time: Upper plot shows the numerical solution (solid line) compared with the perturbation solutions $\left(\mathcal{O}(\epsilon)\right.$ is dotted, $\mathcal{O}\left(\epsilon^{2}\right)$ is dot-dashed and $\mathcal{O}\left(\epsilon^{3}\right)$ is dashed). Lower plot shows comparison of numerical solution (solid line) and the logarithmic solution (dashed). 
to slow the movement down. The $x$ solution is more interesting, it demonstrates a leading order effect from the drag coefficient $k_{l}$, the angle $\gamma$ and the velocity $v$. So, for a given kick, fixed $\gamma, v$ it is clear that the motion varies solely though the effect of the drag coefficients.

\section{Conclusions}

Now we return to the question of whether the ball can provide an advantage for a team. In the case of Bidvest Wits the perturbation solution can be used to find a clear answer. The swerve in the $x$ direction is proportional to $k_{l}=\rho A C_{l} /(2 m)$ and the value of the air density $\rho$ decreases with altitude (and other atmospheric conditions) while $C_{l}$ increases with the ball roughness. In Johannesburg, at an altitude of $1800 \mathrm{~m}, \rho \approx 1.04 \mathrm{~kg} / \mathrm{m}^{3}$ is approximately $20 \%$ lower than at sea-level, $\rho \approx 1.29 \mathrm{~kg} / \mathrm{m}^{3}$. Hence a team accustomed to playing at sea level will expect approximately $20 \%$ more swerve. In Figure 4 we show solutions for two identical kicks taken at sea level and at $1800 \mathrm{~m}$. Unlike previous solutions we now impose an initial velocity in the $x$ direction such that the ball just enters the goal (for the sea-level kick) situated around $17 \mathrm{~m}$ away. The same kick in Johannesburg would miss by over $30 \mathrm{~cm}$.

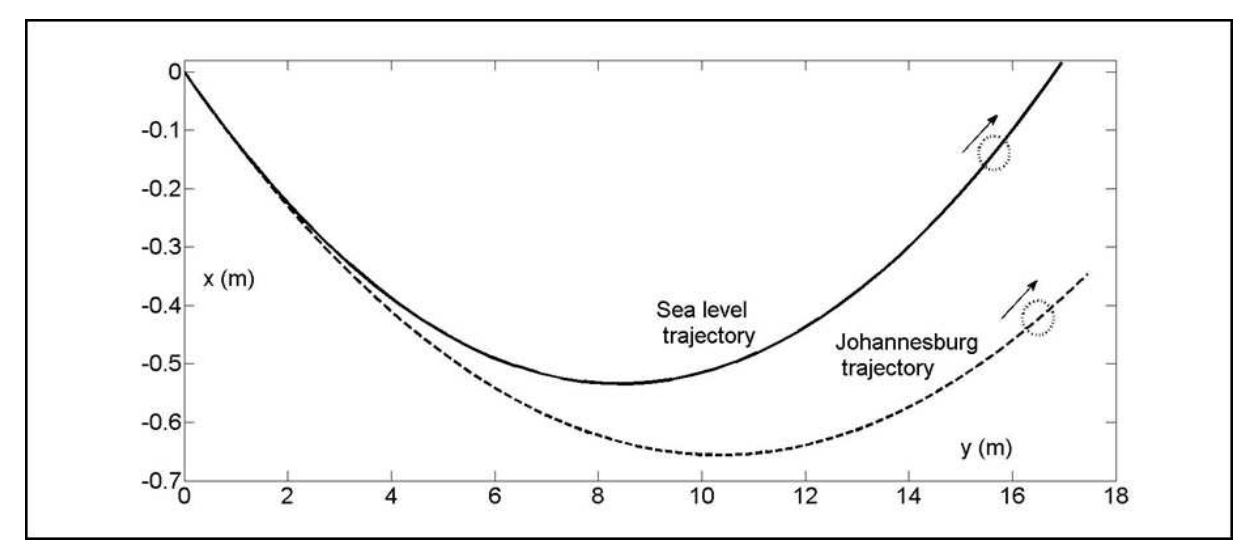

Figure 4: Comparison of identical kicks at sea-level and Johannesburg.

Given that coastal teams expect more swerve the strategy for Wits should be to use a smooth ball (which has a lower $C_{l}$ value) since this will behave in the manner furthest to that expected by a lower altitude team. When training for games at lower 
altitude, a rough ball should be used such that $C_{l}$ increases in a way to reproduce the value of $k_{l}$ expected at the away game (of course this may be difficult to predict). Since $k_{l} \propto C_{l} \rho$ if we increase the value of $C_{l}$ by a factor $1.29 / 1.04$ the kick in Johannesburg will resemble that at sea-level.

We have now submitted a paper to the Journal of Sports Science which expands on the work conducted at the study group and summarised in this report [6].

\section{References}

[1] Bray, K. and Kerwin, D.. Modelling the flight of a soccer ball in a direct free kick, J. Sports Sci., 21 (2003), 75-85.

[2] Dupeux, G., Le Goff, A., Quéré, D. and Clanet, C. The spinning ball spiral, New J. Phys., 12 (2010), 093004.

[3] Goff, J.E. and Carré, M.J. Trajectory analysis of a soccer ball, Am. J. Phys., 77 (2009), 1020-1027.

[4] Griffiths, I., Evans, C. and Griffiths, N. Tracking the flight of a spinning football in three dimensions, Meas. Sci. Technol., 16 (2005), 2056-2065.

[5] Horzer, S., Fuchs, C., Gastinger, R., Sabo, A., Mehnem, L., Martinek,J. and Reichel, M. Simulation of spinning soccer ball trajectories influenced by altitude, 8th Conference of the Int. Sports Engng. Assoc. (ISEA), Procedia Engng. 2 (2010), 2461-2466.

[6] Myers, T.G. and Mitchell, S.L. How to choose a soccer ball (and confuse your enemies), submitted to J. Sports Sci., May 2011.

[7] Oggiano, L. and Sætran, L. Aerodynamics of modern soccer balls, Procedia Engng, 2 (2010), 2473-2479. 